This item was submitted to Loughborough's Research Repository by the author.

Items in Figshare are protected by copyright, with all rights reserved, unless otherwise indicated.

\title{
Improved model for circumsolar irradiance calculation as an extended light source and spectral implications for high-concentration photovoltaic devices
}

PLEASE CITE THE PUBLISHED VERSION

http://dx.doi.org/10.1109/JPHOTOV.2015.2493362

PUBLISHER

IEEE

VERSION

VoR (Version of Record)

\section{PUBLISHER STATEMENT}

This work is made available according to the conditions of the Creative Commons Attribution 3.0 Unported (CC BY 3.0) licence. Full details of this licence are available at: http://creativecommons.org/licenses/by/3.0/

LICENCE

CC BY 3.0

\section{REPOSITORY RECORD}

Cole, Ian R., and Ralph Gottschalg. 2019. "Improved Model for Circumsolar Irradiance Calculation as an Extended Light Source and Spectral Implications for High-concentration Photovoltaic Devices”. figshare. https://hdl.handle.net/2134/19817. 


\title{
Improved Model for Circumsolar Irradiance Calculation as an Extended Light Source and Spectral Implications for High-Concentration Photovoltaic Devices
}

\author{
Ian Richard Cole and Ralph Gottschalg
}

\begin{abstract}
With concentrator photovoltaics (CPV) technology soon to enter a phase of further development in the USA Sunbelt region, it is imperative to update the predictive tools associated with the technology. This involves modeling the Sun as an extended light source with particular attention to circumsolar irradiance. An improvement to the standard extended light source solar profile model is presented based on an improved parameterization of the circumsolar irradiance. A case study for a solar profile pertaining to a circumsolar ratio (CSR) of 0.3 shows incident direct normal irradiance collection overestimations in the standard model of $0.5 \%-1.5 \%$. The model presented here mitigates these errors and corrects for a misrepresentation of CSR in the standard model. For an input CSR resolution of 0.01 , the mean and standard deviation of normalized output CSR are improved from 0.937 and 0.107 to 1.000 and 0.005 . Furthermore, a model extension is presented, incorporating the spectral distribution differences in the central solar and circumsolar regions. A banded spectral analysis of CSR and air mass variation effects are presented, corresponding to the subcells of the multijunction cell architecture. Significant differences are found in the trends of each subcell regarding the relationship between these variables and usable input irradiance.
\end{abstract}

Index Terms-Circumsolar ratio (CSR), concentrator photovoltaics (CPV), irradiation harvest, irradiance modeling solar profile.

\section{INTRODUCTION}

$\mathbf{S}^{\circ}$ OLAR irradiance is typically described as a point source and the circumsolar region is often not considered. The point source approximation is entirely sufficient to model the majority of solar energy systems. However, the onset of concentrated solar power, particularly high-concentration photovoltaics (HCPV), requires revisiting these approximations for applications where the optical input aperture angle approaches that subtended by the solar disk. This resulted in the development of spatially distributed solar flux measurements and derived pre-

Manuscript received August 11, 2014; revised October 31, 2014, and October 6, 2015; accepted October 13, 2015. Date of publication November 11, 2015; date of current version December 18, 2015. This work was supported by a joint U.K.-India initiative in solar energy through a joint project "Stability and Performance of Photovoltaics" funded by Research Councils UK Energy Program in the U.K. under Contract EP/H040331/1 and by the Department of Science and Technology in India.

The authors are with the Centre for Renewable Energy Systems Technology, Wolfson School, Loughborough University, Loughborough LE11 3TU, U.K. (e-mail: I.R.Cole@lboro.ac.uk; R.Gottschalg@lboro.ac.uk).

Color versions of one or more of the figures in this paper are available online at http://ieeexplore.ieee.org.

Digital Object Identifier 10.1109/JPHOTOV.2015.2493362 dictive models [1], [2]. These require treating the Sun as an extended light source as this allows for more accurate ray trace modeling and the advancement of optical system design for CPV applications.

CPV has the potential to reduce the cost of PV [3]-[7] but the commercialization of $\mathrm{CPV}$ has been significantly slower than that of the flat plate PV. Its current share of the PV market is much less than 1\% [8]. Improved accuracy of the available modeling tools will aid the development and deployment of this technology as uncertainties in the modeling are associated with increased financial risk (and, thus, higher financing costs).

Point source solar irradiance models and solar disk containment models such as pillbox or Gaussian distributions lead to overestimations in the CPV system performance [9], [10]. Extended light source models incorporating atmospheric paraxial refractions surrounding the solar disk and the formation of the solar aureole [11] better approximate the solar resource and improve the CPV energy yield models, although the inherent overestimation problems still remain in some of these approaches. This paper identifies and addresses the systematic overestimation in CPV optical modeling by means of an improved model for circumsolar irradiance calculation as an extended light source.

\section{Circumsolar Ratio Modeling}

The current standard model for the description of circumsolar irradiation is the circumsolar ratio (CSR) model developed by Buie et al. [1], hereafter referred to as the Buie model. The model generates a 1-D intensity distribution describing power as a function of angular deviation from the solar center for a given CSR. CSR variation differs with terrestrial location. There is currently little data available on the location-dependent CSR variations, although there are now established measurement efforts to this end [2], [12]. Such data will prove valuable in CPV system performance predictions. The data on which the Buie model is based were recorded by the Lawrence Berkley Laboratory (LBL) in the 1970s and is freely available in the LBL reduced database [13].

The CSR is defined as the ratio of power in the circumsolar region to net power in the central and circumsolar regions combined. Thus

$$
C=\frac{\int_{\theta_{\text {Sun }}}^{\theta_{\text {Circumsolar }}} \varphi(\theta) d \theta}{\int_{0}^{\theta_{\text {Circumsolar }}} \varphi(\theta) d \theta}
$$


where $C$ denotes the CSR, $\varphi$ denotes the solar flux intensity, $\theta$ denotes the angular deviation from the solar center, $\theta_{\text {Circumsolar }}$ denotes the angular limit of the circumsolar region, and $\theta_{\text {Sun }}$ denotes the angular limit of the central solar disc.

The central solar region is essentially the effective size of the Sun due to the Earth-Sun geometry. Measurement of the absolute astronomical unit (au) subtended radial half angle of the solar center is a nontrivial task. Depending on the location of measurement, measurement technique, and measurement time, one will find small variations in the subtended solar semidiameter [14].

In the modern measurement studies, subtended radial solar half angle averages tend to be quoted around $959^{\prime \prime}$ or 4.65 mrad [15], [16], which coincides with the average apparent solar semidiameter used by the Buie model.

The apparent solar semidiameter changes with the Sun-Earth distance according to

$$
\delta=\tan ^{-1}\left(\frac{d}{2 D}\right)
$$

where $\delta$ is the apparent solar semidiameter, $d$ is the diameter of the Sun, and $D$ is the Sun-Earth distance.

Due to the elliptical orbit of the Earth about the Sun, there is a $\sim 3 \%$ variation in the apparent solar semidiameter throughout the year from perihelion to aphelion of 4.742 to $4.584 \mathrm{mrad}$, respectively. Good arguments exist for the inclusion of such variations in extended light source modeling [17], namely that calculated CSR changes with defined central solar boundaries.

Beyond the solar disk paraxial refraction and atmospheric scattering contribution to the circumsolar region, the circumsolar region encompasses the solar aureole and extends to the start of the blue sky diffuse radiation region. The exact point at which the solar irradiation can be considered diffuse is arguable and different interpretations exist. The circumsolar region may extend somewhat beyond $43.6 \mathrm{mrad}$, but it is this limit that is used in the Buie model due to the measurement restrictions on the available data in the LBL reduced database [18], [19], on which the model is based. Furthermore, due to the exponential fall off of radiation in the circumsolar region with angular deviation from the solar center, contributions beyond this limit may be considered negligible.

The CSR in the Buie model is calculated from (1) as

$$
C=\frac{\int_{\theta=4.65}^{\theta=43.6} \varphi(\theta) d \theta}{\int_{\theta=0}^{\theta=43.6} \varphi(\theta) d \theta}
$$

where $\theta$ is the angular deviation from the solar center in mrad.

The Buie model establishes the following relationships:

$$
\begin{aligned}
\varphi_{\text {norm }}(\theta) & = \begin{cases}\frac{\cos (0.326 \theta)}{\cos (0.308 \theta)}, & 0 \leq \theta \leq 4.65 \mathrm{mrad} \\
e^{\kappa} \theta^{\gamma}, 4.65 \mathrm{mrad} & <\theta \leq 43.6 \mathrm{mrad}\end{cases} \\
\kappa & =0.9 \ln (13.5 C) C^{-0.3} \\
\gamma & =2.2 \ln (0.52 C) C^{0.43}-0.1
\end{aligned}
$$

where $\varphi_{\text {norm }}$ is the normalized solar flux intensity.
Assuming rotational symmetry, one can utilize this model to develop an intensity distribution as a function of azimuth and elevation angle coordinate pairs. The resultant extended light source distribution facilitates 3-D ray trace simulations and, hence, offers analytical insights into the CPV optical system design.

The Buie model forms the basis of this investigation. This model is empirically derived from a single database of CSR measurements and as such could be considered somewhat biased. However, the analytical methods presented herein can be applied to any solar irradiance distribution model that operates under the assumption of a rotational symmetry.

Although it is known that the CSR variation can impact the CPV system performance, data on location-specific CSR variation are sparse. A probabilistic framework for CSR variation based on the existing datasets has been used in previous works [10], although it is recognized that this is not an ideal approach. Further, CSR measurement campaigns [2], [12] are essential for the advancement of small entry aperture, high concentration $\mathrm{CPV}$, and CSP system modeling. The complexity of CSR variability, in particular its relationship to aerosol particles and the chaotic variability of the Earth's atmosphere [20], means that circumsolar radiation effects are terrestrially ubiquitous. Consideration of this parameter is, thus, important independent of terrestrial location, although time-averaged data for CSR variation from further measurements will provide extra insight as to terrestrial variation and regions of particular sensitivity.

\section{EXTENDED Light SOURCE Sunshapes}

A volumetric analysis of extended light source sunshapes over the beam irradiation incident angle range allows for an investigation of relative intensity contributions confined to specified areas of sky. Hence, the sunshapes presented herein are displayed in the volumetric form.

Equations (4)-(6) have here been used to generate an extended light source solar profile for a characteristic CSR of 0.3 . This CSR was chosen as it is particularly interesting for CPV-an occurring solar profile that is rarely considered in the CPV optical system design. The resultant intensity profile as a function of azimuth and elevation angle as generated by the application of rotational symmetry is shown in Fig. 1.

Fig. 1 shows a surface plot of 1 million random homogeneously distributed mesh points over the full solar profile region as generated by the model. The spatially biregional nature of solar irradiation is clearly visible here, namely in the differentiation between central solar and circumsolar regions. A significant drop in radiation intensity can be seen at the transition from the central solar region to the circumsolar region. The circumsolar region is volumetrically significant despite the sharp intensity drop. The area contribution of the circumsolar region is much larger than that of the central solar region.

It is possible to check this model by means of geometric interpretation. By considering the solid angle within which the model operates as a circular boundary in an $x y$ plane, and the power at a given angular coordinate pair as an associated $z$ value, 


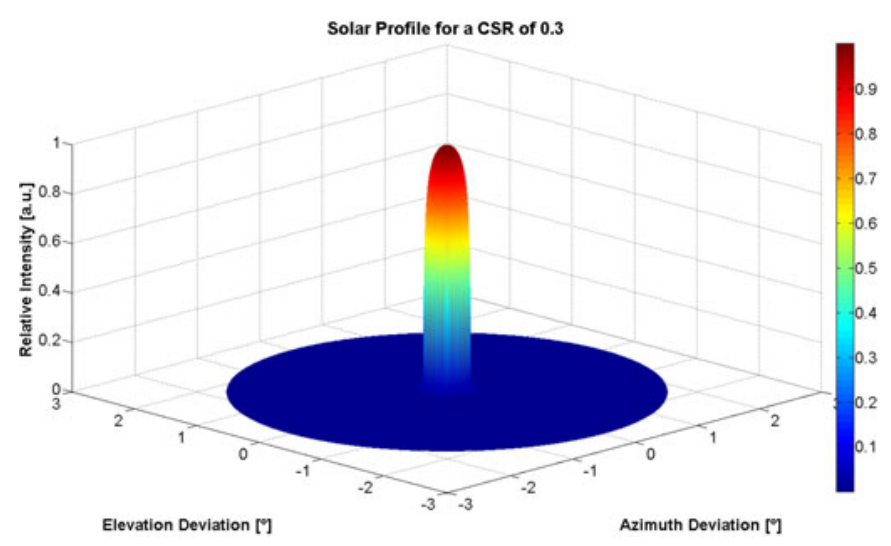

Fig. 1. Three-dimensional solar profile for an input CSR of 0.3. The central solar and circumsolar regions are clearly visible. The major portion of irradiance is contained within the central solar region. The circumsolar region extends far beyond the central solar region.

the net power within a region of space is computed as a volume integral over the correspondingly confined $x y$ space.

By definition, for the above solar profile, the ratio of volume over the circumsolar region, as defined in (7), to volume over the central solar region, as defined in (8), should be 0.3 . However, the actual model output is around $9 \%$ lower than this (see Table I, which has been provided as supplementary material). In [21] and [22], the model authors note these discrepancies and interpret them as deviations in the model's prediction of the edge of the solar disk. However, it is apparent from (4) that the edge of the solar disk is inherently defined by the model as $4.65 \mathrm{mrad}$, which is the average apparent solar semidiameter. Moreover, the projected deviations in effective solar disk size fall beyond the physical boundaries defined in (2). An alternative approach to the model correction is advocated here by means of an input CSR correction. This facilitates output smoothing without the need for unphysical assertions. Empirical correction formulas have been derived, although given their complexity, a correction table is also provided as an appendix

$$
\begin{aligned}
& D_{\text {Circumsolar }}=\sqrt{x^{2}+y^{2}}>4.65 \mathrm{mrad} \\
& D_{\text {CentralSolar }}=\sqrt{x^{2}+y^{2}} \leq 4.65 \mathrm{mrad}
\end{aligned}
$$

where $D_{\text {Circumsolar }}$ is the circumsolar region, $D_{\text {Centralsolar }}$ is the central region, $x$ is the azimuth angular deviation from the solar center, and $y$ is the elevation angular deviation from the solar center.

The central solar and circumsolar regions, as defined in (6), for an input CSR of 0.3 are presented separately in Figs. 2 and 3.

Fig. 2 shows a surface plot of 1 million random homogeneously distributed mesh points over the central solar region. There is a very slight slope of decreasing intensity in the center with an increase in intensity drop with angular deviation toward the circumsolar region. The resultant volumetric surface is a thimble-shaped distribution. According to the model, the normalized central solar profile is invariant under changes in CSR.

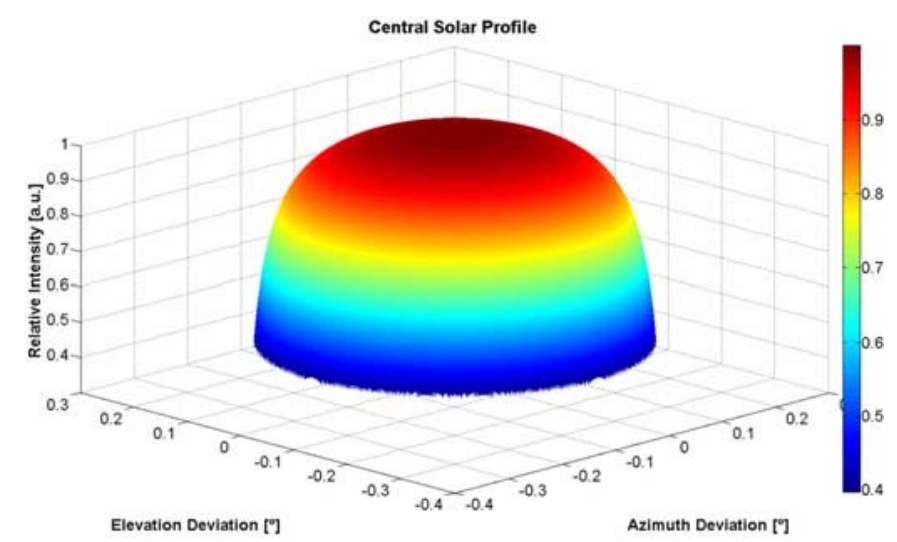

Fig. 2. Central solar region intensity distribution, normalized to a maximum intensity of 1 . The enveloped surface is near-cylindrical at center with a significant falloff with angular deviation.

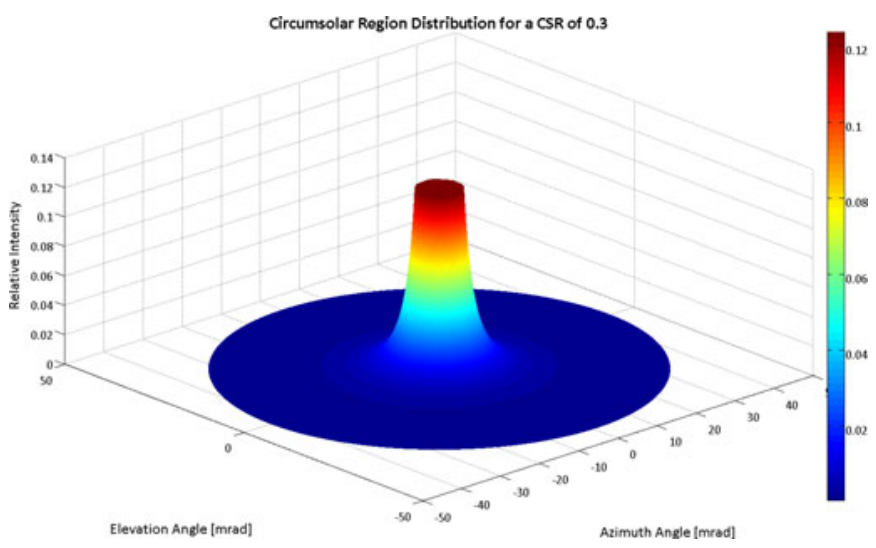

Fig. 3. Circumsolar region intensity distribution for an input CSR of 0.3 , normalized to a maximum intensity of 1 . If the intensity were in $\log$ space, the enveloped shape would be a rotated trapezium.

Fig. 3 shows a surface plot of 1 million random homogeneously distributed mesh points over the circumsolar region. The logarithmic intensity decrease with radial distance is clearly visible.

By comparing the volumes of each region as generated by the model, the output and input CSRs can be compared. Numerical analysis is used here for volume calculation; a potential benefit of this method is that it facilitates the investigation of averaging effects due to angular resolution. The process is outlined in the following section.

\section{VOLUMETRIC ANALYSIS}

The region volumes can be calculated as triple integrals according to

$$
V=\iint d A \cdot d \varphi
$$

where $A$ is the area or solid angle, and $\phi$ is the power.

In the following section, the volumes are calculated by the means of numerical analysis by geometric interpretation. 


\section{A. Central Solar Region}

The area contribution for a given annular central solar region $i$ is found as

$$
A_{i}=\pi(\sin (i \Delta \theta))^{2}-\pi(\sin (i \Delta \theta-\Delta \theta))^{2} .
$$

The central angular deviation reference point for the region is found as

$$
\Theta=i \Delta \theta-\frac{\Delta \theta}{2} .
$$

The volume contribution for the region is then found from (4) as

$$
V_{i}=A_{i} \frac{\cos (0.326 \Theta)}{\cos (0.308 \Theta)}
$$

The angular step in the numerical analysis is defined by the resolution $n$ as

$$
\Delta \theta=\frac{4.65}{n} .
$$

The total volume of the central solar region is then

$$
\begin{aligned}
V= & \sum_{i=1}^{i=n} V_{i}=\sum_{i=1}^{i=n} A_{i} \frac{\cos (0.326 \Theta)}{\cos (0.308 \Theta)}=\sum_{i=1}^{i=n} \pi(\sin (i \Delta \theta))^{2} \\
& -\pi(\sin (i \Delta \theta-\Delta \theta))^{2} \frac{\cos \left(0.326\left(i \Delta \theta-\frac{\Delta \theta}{2}\right)\right)}{\cos \left(0.308\left(i \Delta \theta-\frac{\Delta \theta}{2}\right)\right)}
\end{aligned}
$$

where $n$ is large.

\section{B. Circumsolar Region}

The area contribution for a given annular circumsolar region $i$ is found as

$$
A_{i}=\pi\left((\sin (4.65+i \Delta \theta))^{2}-(\sin (4.65+i \Delta \theta-\Delta \theta))^{2}\right) .
$$

The central angular deviation reference point for the region is found as

$$
\Theta=4.65+i \Delta \theta-\frac{\Delta \theta}{2} .
$$

The volume contribution for the region is then found using (4)-(6) as

$$
V_{i}=A_{i} e^{\kappa} \Theta^{\gamma} .
$$

The angular step in the numerical analysis is defined by the resolution $n$ as

$$
\Delta \theta=\frac{43.6-4.65}{n} .
$$

The total volume of the circumsolar region is then

$$
\begin{aligned}
V= & \sum_{i=1}^{i=n} V_{i}=\sum_{i=1}^{i=n} A_{i} e^{\kappa} \Theta^{\gamma}=\sum_{i=1}^{i=n} \pi\left((\sin (4.65+i \Delta \theta))^{2}\right. \\
& \left.-(\sin (4.65+i \Delta \theta-\Delta \theta))^{2}\right) e^{0.9 \ln (13.5 C) C^{-0.3}} \\
& \left(4.65+i \Delta \theta-\frac{\Delta \theta}{2}\right)^{2.2 \ln (0.52 C) C^{0.43}-1}
\end{aligned}
$$

where $n$ is large.

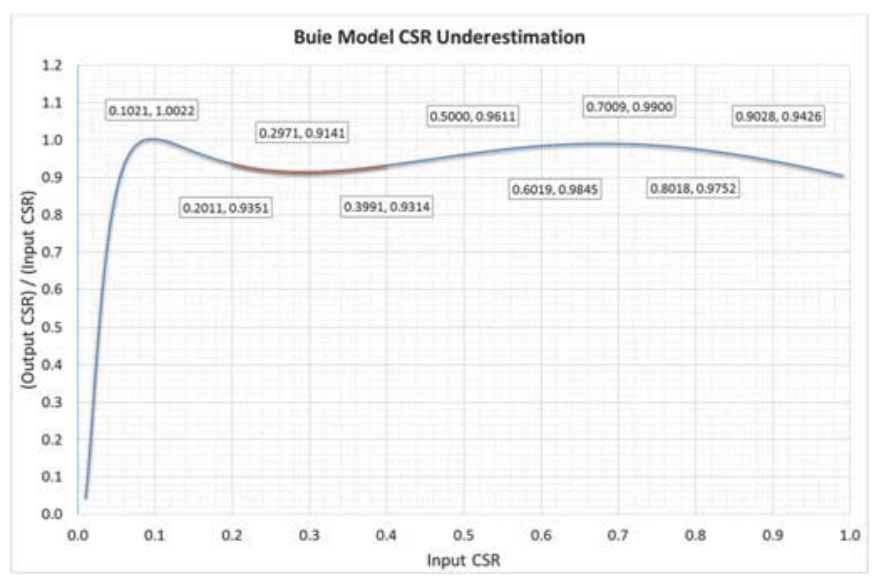

Fig. 4. Buie model output CSR as a proportion of input CSR. The 0.2 to 0.4 CSR region is highlighted as a particular region of interest for CPV optical system performance.

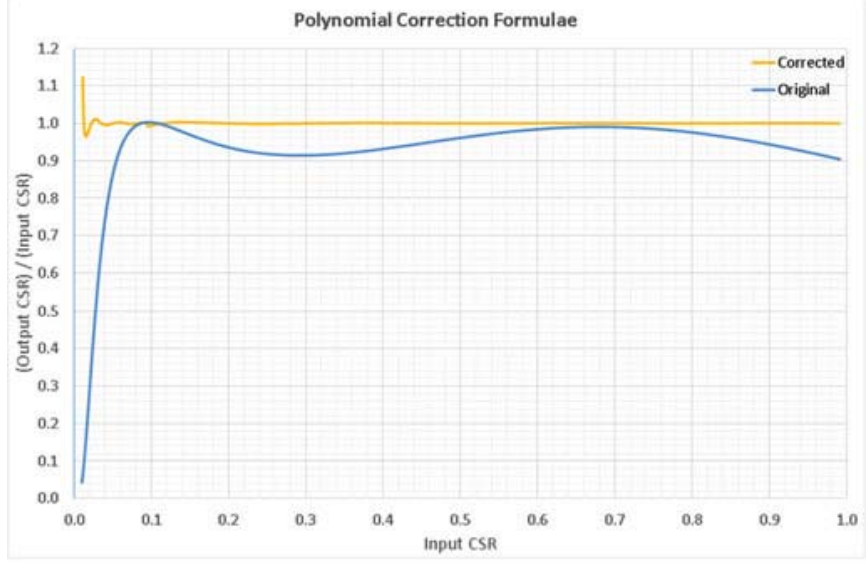

Fig. 5. Polynomial formulae correction comparison with original. A near 1:1 input-output ratio is found when using the correction formulae.

In this paper, a value of 10000 is used for $n$ as this satisfies convergence to three decimal places.

\section{Circumsolar Ratio Modeling}

The CSR is a useful parameter for the solar concentration system design and performance modeling as it describes the distribution of irradiance. The Buie model uses this ratio as an input parameter in the generation of a spatially distributed irradiation profile. However, the model suffers from some inherent distortions such that the input CSR parameter differs from the output distribution CSR. This limits the usefulness of the model, particularly in the optical system design process. These deviations are investigated here.

The output CSR calculated from the Buie model is compared with input CSR in Figs. 4 and 5. The CSR range 0.2-0.4 is highlighted as this range is of particular interest for CPV, representing high but occurring CSR values for which extended light source models are required for realistic system performance predictions.

Fig. 4 shows that the Buie model generally underestimates CSR. In the CSR region of 0.1 to 0.6 , the average 
underestimation is $\sim 5 \%$. Moreover, beyond a CSR of 0.1 , the maximal underestimation of $\sim 9 \%$ is found in a particular region of interest for the CPV system modeling. For an input CSR resolution of 0.01 , the mean and standard deviation of the output CSR are 0.937 and 0.107 , respectively.

It is possible to calculate a correction, which will then remove this systematic underestimation. These correction formulas have been calculated by polynomial fitting to adjust to a near $1: 1$ input-output ratio. These are presented as a bisectional analysis

$$
\begin{aligned}
& \mathrm{CSR}_{I}= \\
& \begin{cases}14.66 C^{6}-42.315 C^{5} & \\
+49.513 C^{4}-28.761 C^{3}, & 0<C<0.096 \\
+8.0542 C^{2}+0.137 C+0.0291 & \\
-915401 C^{6}+293962 C^{5} & \\
-37145 C^{4}+2355.9 C^{3}, & 0.096<C<1 \\
-77.581 C^{2}+1.981 C+0.0104 & \end{cases}
\end{aligned}
$$

where $\mathrm{CSR}_{I}$ denotes the input CSR, and $C$ denotes the desired CSR.

Using these polynomials to correct the input CSR in the original model, the output/input ratios approach unity as shown in Fig. 5.

Fig. 5 shows the model improvement by input CSR correction according to (20). For an input CSR resolution of 0.01 , the mean and standard deviations of the output CSR are 1.000 and 0.005 , respectively. A clear improvement can be seen. The correction formulas can obviously be further improved in the near-zero input CSR region, although the above will be sufficient for the majority of all applications. The near-zero region is less critical as the impact of circumsolar radiation is relatively insignificant.

Given that the resultant formulas are far from elegant, a correction table is appended for reference. The table provides a set of reference points for the input CSR corrections as generated by (20). The resolution of appended reference table should prove sufficient for most cases.

Extended light source Sun models are particularly useful for the investigation of sunshape-specific optical effects. The regional dependence of spectral effects is of particular importance in CPV modeling (see Section VII). Given this, it is important that any CSR-specific phenomena or conclusions inferred from these models reference the correct CSR. The correction formula presented here serves to address discrepancies to this end in a popular existing model.

\section{IMPROVED IRRADIANCE HARVEST CALCULATION TEST CASE}

Irradiance collection is defined here as that irradiance visible to the primary concentrator, i.e., within the bounds of the primary concentrator lens aperture. It is this irradiance that can be harvested by the concentration system. The irradiance harvest is most notably a function of the optical system input aperture,

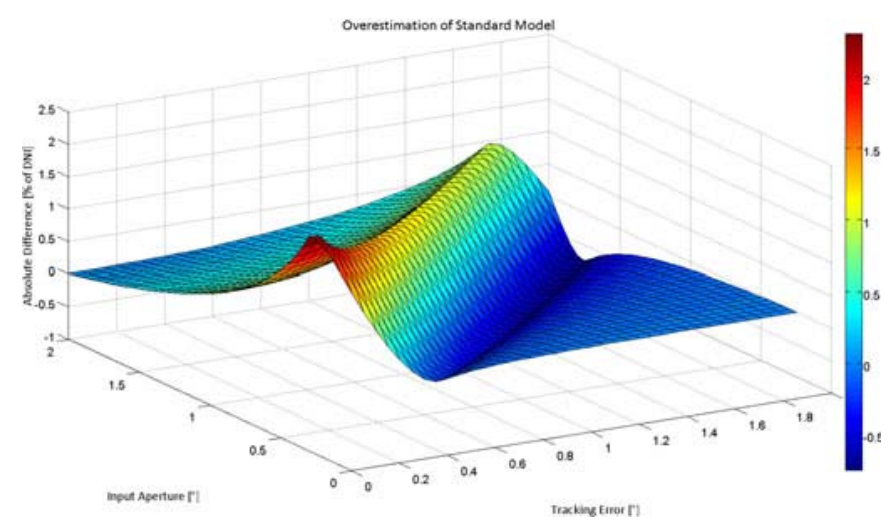

Fig. 6. Model irradiation harvest overestimation surface plot for an input CSR of 0.3 . Overestimation is given in terms of absolute \% of DNI versus optical system input aperture versus tracking error.

the solar tracking error, and the distribution of irradiation, as characterized by the CSR.

The irradiance collection versus tracking error versus halfangle input aperture has been calculated using both the original model and the corrected case. A CSR of 0.3 was chosen for analysis and a corrected input CSR of 0.3274 was derived from the appended correction table. Fig. 6 shows the overestimation of the standard model in terms of absolute percentage of DNI.

Fig. 6 shows the regions of both overestimation and underestimation of irradiation collection by the standard model for an input CSR of 0.3, with overestimation dominating. An irradiation harvest overestimation of $\sim 2.3 \%$ of absolute DNI is found with an input aperture of $0.25^{\circ}$ and ideal solar tracking. For a given input aperture, maximum overestimation is found at $\sim T=A-\theta_{\text {Sun }}$ and maximum underestimation at $\sim T=A+\theta_{\text {Sun }}$. These threshold values are the same for any distribution with underestimated circumsolar contributions, although the space between varies.

\section{MODEL EXTENSION FOR SPECTRAL CONSIDERATIONS}

The spectral sensitivity of multi-junction (MJ) solar cells is an issue of much concern for CPV systems [23]-[25]. An MJ cell is essentially a series connected set of single-junction solar cells, each with a different bandgap and, thus, responding to a different portion of the incident solar spectrum. The net power produced by a series connected set of electrical generators is limited by the electrical mismatch of the components according to

$$
P=I_{\min } \sum^{V}
$$

where $P$ denotes the output power, $I_{\min }$ is the minimum operational current of the device set, and $V$ is the device voltages.

This phenomenon is known as current mismatch. Current mismatch is an inherent feature of the MJ solar cell. MJ cell structures are designed for the optimum performance under specific spectra such as the AM1.5D spectrum. The default AM1.5D spectrum, as generated by SMARTS, has a CSR of less than 0.01 , which is notably small. However, actual 


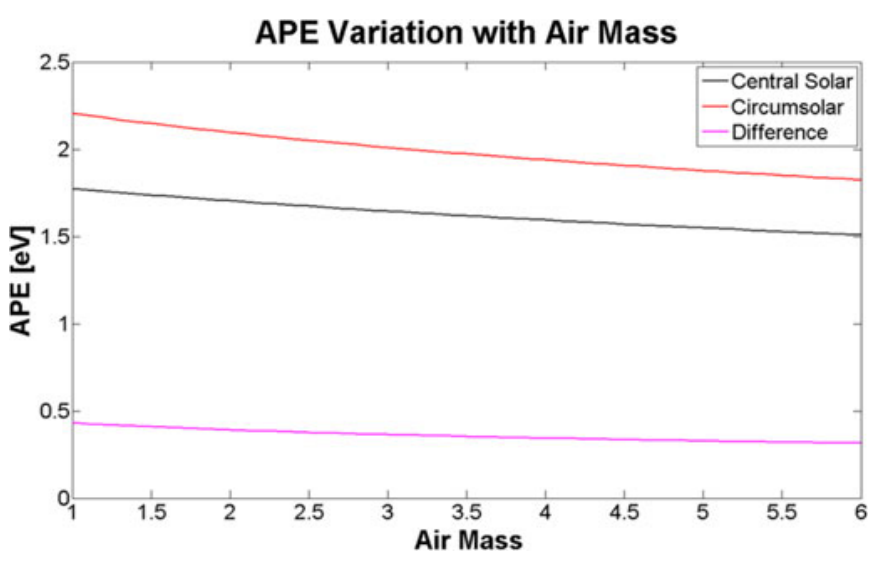

Fig. 7. APE variation with air mass for the central solar and circumsolar regions.

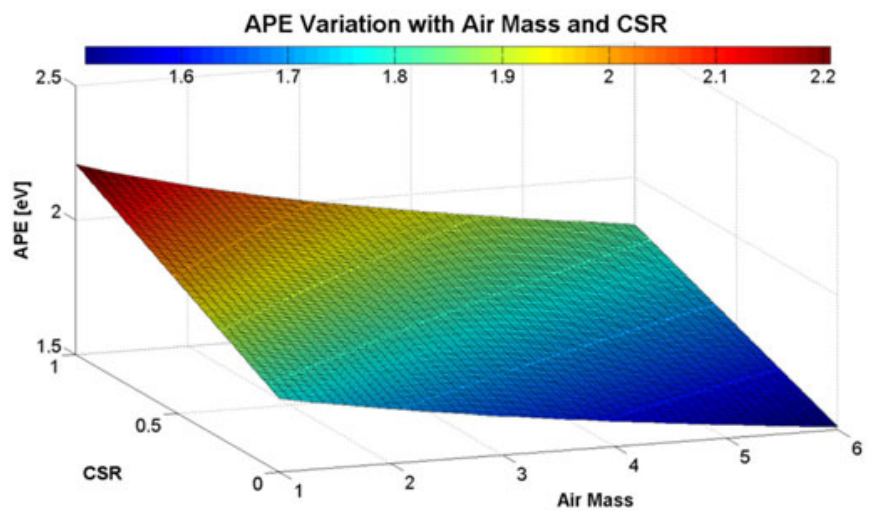

Fig. 8. APE of spectra with variation of air mass and CSR

incident spectrum varies with a large variety of atmospheric parameters. In particular, the CSR is notably significantly affected by aerosols and is in fact the principal error source in well-calibrated measurements of aerosol optical depth [20]. The CSR becomes increasingly interesting when considering its role in the spectral variation. A model extension incorporating the spectral variations associated with the CSR is here presented.

The model presented herein is biregional according to (7) and (8). Due to the different physical mechanisms behind their projection, the central solar and circumsolar regions differ in their spectral compositions. The average photon energy (APE) of these regions is compared over an air mass range of 1 to 6 in Fig. 7. The data were generated using SMARTS by varying the air mass parameter and keeping all other parameters at the default (AM1.5) values. A 280-4000 nm spectral range was used. The APE of the central solar and circumsolar regions for the AM1.5 reference spectrum is 1.74 and $2.15 \mathrm{eV}$, respectively.

These region-specific spectral variations cause a corresponding change in the incident spectrum with CSR, as shown in Fig. 8. The effect is nonlinear, as can be seen from the contour line variations.

The APE is a useful metric for the qualification of spectral variation, although it does somewhat simplify the actual spectral variations, particularly their region-specific nature. This is highlighted in the example of Fig. 9.

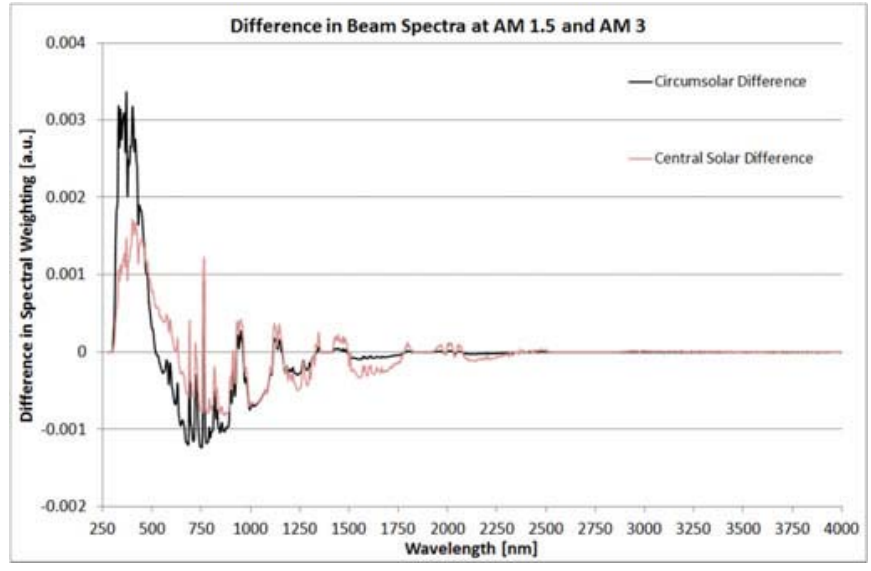

Fig. 9. Difference in normalized spectral weighting at air mass 1.5 and 3 for the central solar and circumsolar regions. The units are chosen such that the integration of the normalized actual spectra across all wavelengths yields 1 .

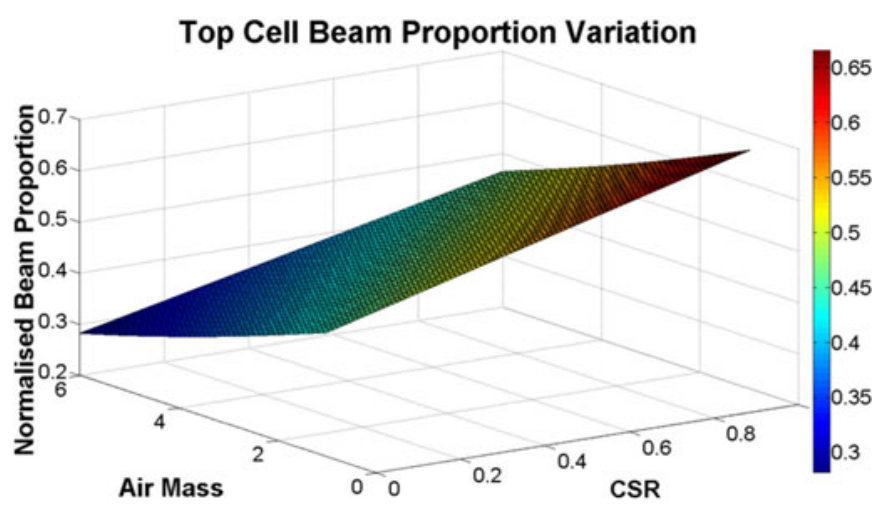

Fig. 10. Top cell $(280-680 \mathrm{~nm})$ spectral band beam proportion versus air mass versus CSR.

The complex spectral dependence of absorption variation is particularly interesting when analyzed in the context of MJ solar cell architecture. An example architecture-specific analysis is given here using a typical triple-junction solar cell. The operational bandwidth ranges of the subcells of a typical triple-junction solar cell optimized for the AM1.5D spectrum are approximately $280-680 \mathrm{~nm}(1.82 \mathrm{eV})$ for the top cell, $680-880 \mathrm{~nm}(1.41 \mathrm{eV})$ for the middle cell, and $880-1880 \mathrm{~nm}$ $(0.66 \mathrm{eV})$ for the bottom cell [26]. As shown in Figs. 10-12, the variations in incident spectra resulting from changes in air mass and CSR alter the proportion of beam irradiation that falls within the active region of each MJ cell. Moreover, the variable dependencies are significantly different in each region, as evidenced by the dramatic difference in trends.

The top cell spectral band beam proportion has a minimum at low CSR and high air mass and a maximum at high CSR and low air mass.

The middle cell spectral band beam proportion has a minimum at high CSR and low air mass and a maximum at high CSR and high air mass.

The bottom cell spectral band beam proportion has a minimum at high CSR and low air mass and a maximum at low CSR and high air mass. 


\section{Middle Cell Beam Proportion Variation}

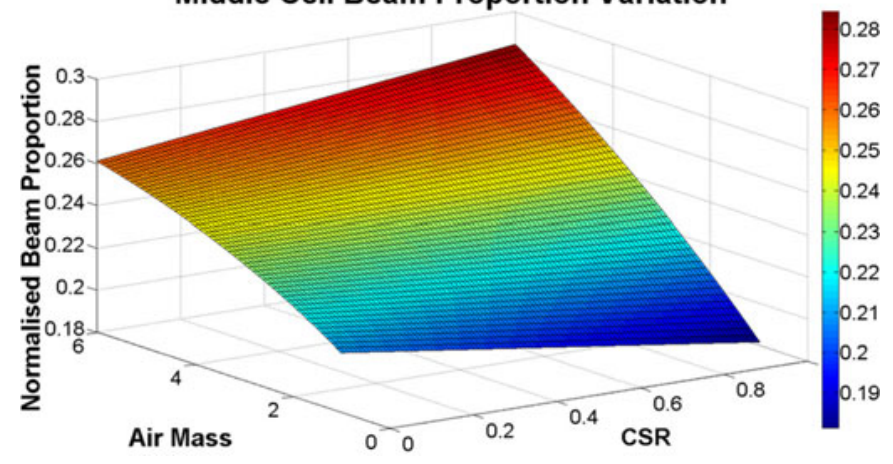

Fig. 11. Middle cell $(680-880 \mathrm{~nm})$ spectral band beam proportion versus air mass versus CSR

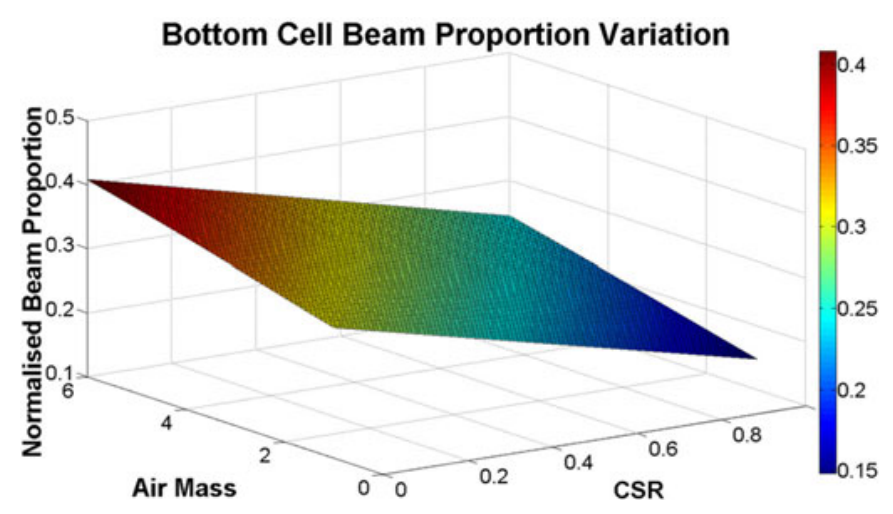

Fig. 12. Bottom cell $(880-1880 \mathrm{~nm})$ spectral band beam proportion versus air mass versus CSR.

The addition of an spatially distributed spectra to an extended light source Sun model is highlighted here as being a particularly important consideration for CPV optical simulations due to potentially depredatory induced power swings in active subcell bandwidths. Spectral variations with CSR are rarely accounted for in CPV optical simulations today. In order to correctly analyze the system performance under the full dynamic range of meteorological uncertainties, solar distribution models must not only better approximate the spatial distribution of solar irradiation but better recreate CSRs as well. This will allow for an improved investigation of specific optical effects relating to CSR variations, which could prove extremely useful in the evaluation of installation site suitability. The model correction proposed in Section V is, thus, further highlighted.

\section{CONCLUSION}

An input/output analysis of the Buie model has been performed and inconsistencies identified. A case study of the $\mathrm{CSR}=0.3$ distribution revealed overestimations of irradiation harvest by the standard model of $0.5 \%-1.5 \%$ of DNI for a system operation region commonplace in HCPV systems. Such overestimations are significant enough to play a depredatory role in CPV energy harvest performance predictions. An extended light source distribution model improvement has been proposed and has been shown to give more consistent CSR output. For an input CSR resolution of 0.01 , the mean and standard deviation of the output CSRs are improved from 0.937 and 0.107 to 1.000 and 0.005 .

Significant trend differences for spectral variations in the spectral bandwidths corresponding to the MJ subcell regions have been identified. For the triple junction cell analysis performed here, the sign of the correlation of input power with CSR and air mass varies across the three junctions. The nature of these junction-specific correlations provides further insight into the difficulties of MJ subcell bandgap optimization under varying conditions.

\section{REFERENCES}

[1] D. Buie, A. Monger, and C. Dey, "Sunshape distributions for terrestrial solar simulations," Sol. Energy, vol. 34, pp. 113-122, 2003.

[2] S. Wilbert et al., "Measurement of solar radiance profiles with the sun and aureole measurement system (SAM)," presented at the SolarPACES Conf., Granada, Spain, 2011.

[3] W. Stolte, "Engineering and economic evaluation of central-station photovoltaic power plants," Electr. Power Res. Inst., Palo Alto, CA, USA, TR-101255, 1992.

[4] M. Symko-Davies, R. Noufi, and S. Kurtz, "Identifying critical pathways to high-performance PV," in Proc. IEEE 29th Photovoltaic Spec. Conf., New Orleans, LA, USA, 2002, pp. 1729-1732.

[5] R. Whisnant, S. Johnston, and J. Hutchby, "Economic analysis and environmental aspects of photovoltaic systems," Handbook of Photovoltaic Science and Engineering, A. Luque, Ed. Chichester, U.K.: Wiley, 2003, pp. 971-1003.

[6] S. Kurtz, A. Lewandowski, and H. Hayden, "Recent progress and future potential for concentrating photovoltaic power systems," Nat. Renewable Energy Lab., Golden, CO, USA, NREL/CP-520-36871, 2004.

[7] R. McConnell, "Concentrator photovoltaic technologies: Review and market prospects," Refocus, vol. 6, pp. 35-39, 2005.

[8] G. Masson et al., "Global market outlook for photovoltaics 2013-2017," Brussels: Eur. Photovoltaic Ind. Assoc., Brussels, Belgium, 2013.

[9] M. Schubnell, "Sunshape and Its influence on the flux distribution in imaging solar concentrators," ASME J. Sol. Energy Eng., vol. 114, pp. 260-266, 1992.

[10] I. Cole, T. Betts, and R. Gottschalg, "Solar profiles and spectral modelling for CPV simulations," IEEE J. Photovoltaics, vol. 2, no. 1, pp. 62-67, Jan. 2012.

[11] R. Goody and Y. Yung, Atmospheric Radiation: Theoretic Basis. Oxford, U.K.: Oxford Univ. Press, 1996.

[12] S. Wilbert, R. Pitz-Paal, and J. Jaus, "Circumsolar radiation and beam irradiance measurements for focusing collectors," presented at the ES1002, Cost Wire Workshop, Roskilde, Denmark, May 22-23, 2012.

[13] (2014, Aug. 8). Lawrence Berkley Lab. Reduced Database [Online]. Available: http://rredc.nrel.gov/solar/pubs/circumsolar/

[14] E. Reis Neto, A. Andrei, J. Penna, E. Jilinski, and S. Puliaev, "Observed variations of the solar diameter in 1998/2000," Sol. Phys., vol. 212, pp. 7-21, 2003.

[15] G. Thuillier, S. Sofia, and M. Haberreiter, "Past, present and future measurements of the solar diameter," Adv. Space Res., vol. 35, pp. 329-340, 2005.

[16] E. G. Jilinski, S. Puliaev, J. L. Penna, A. H. Andrei, and F. Laclare, "Solar diameter observations at observatorio nacional in 1998-1999," Astron. Astrophys. Suppl. Ser., vol. 135, pp. 227-229, 2000.

[17] A. Neumann, A. Witzke, S. Jones, and G. Schmitt, "Representative terrestrial solar brightness profiles," J. Sol. Energy Eng., vol. 124, pp. 198-204, 2002.

[18] D. Grether, A. Hunt, and M. Wahlig, "Results from circumsolar radiation measurements," presented at the Int. Solar Radiation Conf., Winnipeg, MB, Canada, Aug. 1976.

[19] J. Noring, D. Grether, and A. Hunt, "Circumsolar radiation data: The Lawrence Berkeley laboratory reduced data base," Nat. Renewable Energy Lab., Golden, CO, USA, NREL/TP-262-4429, 1991.

[20] M. Kocifaj and C. Gueymard, "Theoretical evaluation of errors in aerosol optical depth retrievals from ground-based direct-sun measurements due to circumsolar and related effects," Atmos. Environ., vol. 45, pp. 1050-1058, 2011.

[21] D. Buie, C. Dey, and S. Bosi, "The effective size of the solar cone for solar concentrating," Sol. Energy, vol. 74, pp. 417-427, 2003. 
[22] D. Buie, "Corrigendum to 'The effective size of the solar cone for solar concentrating systems," Sol. Energy, vol. 79, pp. 568-570, 2005.

[23] T. Betts, C. Jardine, R. Gottschalg, and D. Infield, "Impact of spectral effects on the electrical parameters of multijunction amorphous silicon cells," in Proc. 3rd World Conf. Photovoltaic Energy Convers., Osaka, Japan, 2003, vol. 2, pp. 1756-1759.

[24] C. Eisler, Z. Abrams, M. Sheldon, X. Zhang, and H. Atwater, "Multijunction solar cell efficiencies: Effect of spectral window," Energy Environ. Sci., vol. 7, pp. 3600-3605, 2014

[25] J. Jaus and C. Gueymard, "Generalised spectral performance evaluation of multijunction solar cells using a multicore, parallelized version of SMARTS," presented at the 8th Int. Conf. Concentrating Photovoltaic Syst., Toledo, Spain, 2012.

[26] W. McMahon, S. Kurtz, K. Emery, and M. Young, "Criteria for the design of $\mathrm{GaInP} / \mathrm{GaAs} / \mathrm{Ge}$ triple-junction cells to optimize their performance outdoors," in Proc. IEEE 29th Photovoltaic Spec. Conf., 2003, pp. 931934.

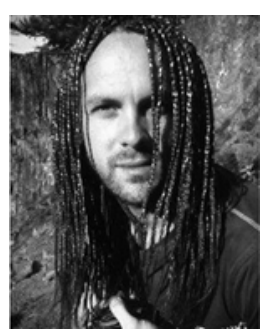

Ian Richard Cole received the B.Sc. degree in physics from Loughborough University, Loughborough, U.K., in 2009 and the Ph.D. degree from the the Centre for Renewable Energy Systems Technology (CREST), Loughborough University, in 2015.

$\mathrm{He}$ is a Research Associate with the Applied Photovoltaics (APV) research group at CREST. His research interests are varied, with specialization in irradiance modelling, distribution and transfer; CPV modelling; system simulations and performance prediction; optical system design and optimization, and modelling parameter optimization and standardization. His current research activities include the harmonization and improvement of PV system modelling standards throughout the European research sector and the advancement of CPV system design and performance prediction methods, with a particular focus on bespoke software development.

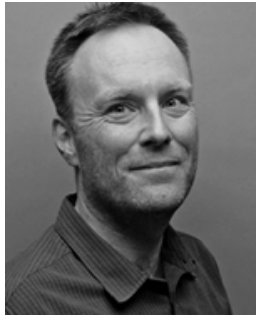

Ralph Gottschalg was born and educated in Karlsruhe, Germany, where he received the Diploma degree in physics from University Karlsruhe and the M.Sc. degree in renewable energy systems technology from Loughborough University, Loughborough, U.K.

He was a Research Engineer with the Energy Department, Fraunhofer Institute for Systems and Innovations Technology, Karlsruhe, before joining Centre for Renewable Energy Systems Technology, in 1996 to pursue the $\mathrm{Ph} . \mathrm{D}$. degree in environmental effects on the performance of photovoltaic devices. He spent his postdoctoral career at Loughborough University, where he held a variety of posts, ultimately achieving the Chair of Applied Photovoltaics. 